\title{
COMMERCIAL POHER FROM INERTIAL CONFINEMENT FUSION: ISSUES AND PROSPECTS
}

Wi?liam Hogan

May 1985

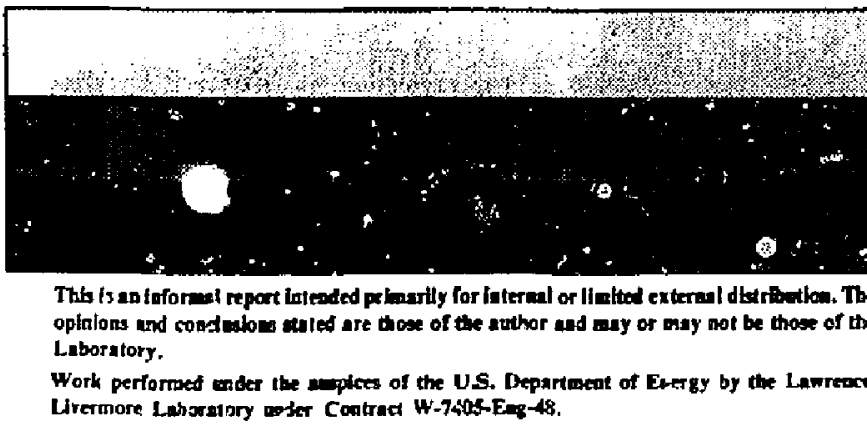

\section{DISCLAIMER}

This report was preparad as an acoount of work sponsored by an agency of the United Slales Government. Neither the Uniled States Government nor any agency thercol, nor any of their cmployes, makes any warranty, cepress or imglied, or assumes ony lesal jiability or responsibility fur the accuracy, complcteness, ar usefulness of any information, apparatus, product, or process disclosed. or represents that its use would nol infringe privately awned rights. Reference herein to any specilic commercia! product, ptocess, or service by trade name, trademark, manufacturer, of otherwise does not necessarily constitute or imply its endorsement, recommendation, or favoring by the United States Govesnment or any agency thereor. The views and opinions of authers expressed herein da nat necessarily state or seflect those of the Uniced States Governtment uf any agencer thereof. 
Commercial Power from Inertial confinement Fusion: I ssues and Prospectild

\section{Executive Summary}

Future criteria for desirable power plants are difficult to determine, but they include economic competitiveness, availability of consumatles, reliability, maintainability, safety, disposal of wastes, and environmental impact. In the near future, the desirability of ICF power clants will probably be evaluated on terms similar to those used for fission reactors, which currently emphasize small, inherently safe reactors constructed from factory-built, standardized modules that can lower the diract capital costs and the construction time.

ICF shares with magnetic fusion many of its desirable features (e.g., inlimited fuel). However, it also has some inherent advantages due to the relaxed vaculiti requirements and the physical separability of the driver, target and reaction chamber. At the same time, some different challenges are presented in the puised nature of its energy and in the manufacture of its targets (fuel pellets).

To be economically competitive with future fission and coal plants, a $1 \mathrm{GW}_{\mathrm{e}}$ ICF power reaci... must have the following characteristics:

Oriver direct cost $\leq \$ 150 / \mathrm{J}$

Driver efficiency $\geq 10 \%$

Target gain $>60-100$ at driver energy of 2-4 MJ

Target factory cost $\leq \$ 100 \mathrm{M}$

Fusion to $\in$ lectric conversion efficiency $\geq 45 \%$

Puise rate $25-10 \mathrm{~Hz}$

plant availability $\geq 0.7$

If these goals are met, the cost of electricity will be iess than $5 t / \mathrm{kW}_{\mathrm{e}} \cdot \mathrm{h}$. Using the same criteria, future fission power may be about 
$3.6 \mathrm{k} / \mathrm{kW}^{-\mathrm{h}}$ and future coal power about $4.7 \mathrm{k} / \mathrm{kW} \mathrm{e}^{\cdot \mathrm{h}}$. If one or more of the above ICF goals is exceeded, then fusion power will be competitive at even smaller piant sizes. ICF is particularly adaptable to the modular construction approach being suggested for fission reactors because of the ability of one driver to support several reactors.

The separability of the driver, target and reaction chamber in ICF power plants has led to a large number of conceptual designs that illustrate the flexibility of ICF technology to meet various relative emphasis among the plant selection criteria. LLNL has recentiy empnasized reactor designs that take advantage of another $1 \mathrm{CF}$ feature, the ability to put large masses of $x$-ray, debris, and neutran absorbing material inside the reaction chamber. In HYLIFE, a thick region of falling liquid lithium jets close to the target reduces the neutron damage in the wall to a level that allows it to survive for the entire 30-year life of the plant. Neutron activation is also greatly reduced compared to the Starfire magnetic fusion reactor design and to PWR's. Pulse*Star uses a LiPb spray and Cascade uses a Li ceramic granule banket to accomplish the same results in addition to eliminating the fire hazard of liquia lithium and raising the permitted pulse rate compared to HYLIFE. These latter two designs also take several steps to improve their economic competitiveness. Pulse*star is a pool-type reactor that has no intermediate heat transfer loop and minimes pipes and valves. Cascade operates at very high temperature, $1200-1700 \mathrm{~K}$, to obtain a high thermal to electric conversion efficiency (55\%). In one version of Cascade, careful selection of materials resulted in further reduction of neutron activation relative to HYLIFE.

For nuclear materials production, the ICF suppressed fission hybrid reactor has several times more neutrons available per unit thermal energy than a rission production reactor. ICF also holds about a $20 \%$ advantage over magnetic fusion for this application. These facts riake the ICF breeder a particularly attractive option for producing tritium, plutonium. 


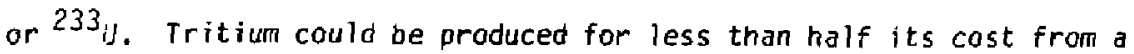
new fission production reactor and an ICF ${ }^{233} \mathrm{U}$ breeder could produce fuel for 18 fission reactors and, thereby, effectively cap fission fuel prices while providing an option for quick response to future fission fuel shortages.

While most ICF reactor issues have been addressed analytically, for many problems, the lack of relevant data is undermining creuibility. ICF is learning a great deal from the MCF reactor technology program. However, those problems that are specific to ICF reactors are receiving almost no experimental attention. Some areas where data and sludies are needed include: neutron danage to reflective optics, dissassembly of isochorically-heated 3 iquid metals, recondensation times for hot plasmas near cold walls, radiation damage for pulsed sources, fracturing of solids in pulsed radiation environments, extraction and recycling of target materials, and target manufacture, injection, and tracking. It is recommended that an ICF reactor technology program be started to address these and other reactor issues through funcamental studies at universities and systems studies in industry with the national laboratories involved in bath. 
Commercial Power from Inertial Confinement Fusion: Issues and Prospects

\section{Introduction}

Among the many existing and potential applications for inertial confinement fusion (ICF) technoiogy, its use as a new source of cominercial power is one of the most promising and, at the same time, challenging goals. ICF shares with magnetic confinement fusion (MCF) the promise of providing a virtually unlimited energy supply from ardinary water, a fuel source that is not dependent on political boundaries. While fusion has its own set of safety arid environmental jssues, they appear to be more tractable than those of many other energy sources. ICF has some inherent advantages over MCF, provided by the relaxed vacuum requirements and the separability of the driver, fusion pellet and reaction chamber. On the other hand, ICF also presents different technological challenges because of the extreme pulsed nature of its energy production and the manufacture and emplacement of its targets (fuei pellets). Thus, while ICF shares many advantages and technological development issues with MCF, it truly represents a very different approach to commercial fusion energy.

Future criteria for determining desirable power plant characteristics are difficult to pin down. It is clear that unless our societal structure for producing power changes dramaticaliy, any new power source must be economically competitive. However, even this very fundamental objective is difficult to use quantitatively because its definition is subject to considerabie disagreement and any given figure of merit is subject to large spacial, temporal, and sociological fluctuations. The course of events in the fission power industry over the last fifteen years provide; ample evidence that perceived economic competitiveness is a necessary but not sufficient condition for a deployment decision on a power source. Other criteria often used explicitly (as well as being folded into the economic evaluation) are availability and reliability of 
the sources of consumables (e.g., fuel); reliability and maintainability of equipment; construction variables such as modularity, expandability. construction time, and technical risk; disposal of wastes; environmental impact; safety; and finally, an extremely hard to define quality, public acceptability. It is important not to overemphasize any one criterion because its relative importance is likeiy to change. The separability of the issues relevant to drivers, targets and reaction chambers provides ICf with a design flexibility that is important in this changing judgmental environment.

While the above arguments imply that we cannat tell what the selection criteria will be 20-40 years in the future, it is useful to examine the criteria that are likely to be used for nearer term, next generation plants because people's willingness to consider fusion as a future source will be colured by those considerations. Recent experience in the fission power industry suggests that there will be a great deal of emphasis on sma11, inherentiy safe reactors made from factory-constructed, standardized modules with as little nuclear grade field construction as possible. In the near term, fusion plant design studies are likely to be evaluated by these same standards.

\section{General Economic Issues}

In order to identify the design and operating space in which ICF will be competitive with fission and coal plants, we have constructed a simple economic model for ICF plants that use a conventional steam cycle. Fission power plants recently completed, and currently under construction, are significantly more expensive than coal-fired plants. Researchers at Oak Ridge National Laboratory ${ }^{\top}$ and United Engineers and Constructors ${ }^{2}$ have estimuted the cost of fuiure (1990's) fission power plants based on the presumed effects of licensing and construction reforms and the experience of the best current power plants. They have 
also estimated costs of future coal plants including flue-gas desulfurization equipment, but no other effluent controls.

Our model for a fusion plant assumes that, other than the driver, target factory and reaction chamber, the rest of power plant will look essentially like a fission power plant outside the fission core (or for that matter, like a coal plant outside the coal combustor). Costs and scaling relationships for these portions of the plant and all the economic factors are thus derived directly from the two referenced studies. Only about $18 \%$ of the direct capital costs of a future PHR are associated with the nuclear steam supply system (NSSS), and only about half of that is associated with the reactor vessel itself. Since this is such a small portion of the total, we make the further simplifying assumption that the fusion reaction chamber cost and scailing are the same as those of a fission reactor vesse (not unreasonable based on cost estimates in recent design studies). Thus, the totat capital cost of in ICF plant in this model would exceed that of a PWR by the costs of the driver and target factory.

The specific cost relationship used for the total capital costs $\left(C_{T O T}\right)$ in January 1983 dollars is:

$$
\mathcal{L}_{\mathrm{TOT}}=\mathcal{L}_{\mathrm{f}}\left[0.705 \mathrm{P}_{\mathrm{g}}^{0.6}+0.363 \mathrm{P}_{\mathrm{g}}^{0.4}+0.15+0.23 \mathrm{E}_{\mathrm{d}}^{0.8}\right](\$ B),(1)
$$

where $C_{1}$ is a factor that accounts for contibiyency and interest during construction $P_{g}$ is the gross electric power $\left(G W_{e}\right)$ and $E_{d}$ is the driver energy (MJ). The first term in equation (1) is the direct capital cost of the reactor ( $i . e .$, the reaction chamber and the balance of plant), the second term is che reactor's indirect cast, the third term is a constant amouni (botin direct and indirect costs) used for the target factory, and the final term is a reasonable cost goal for a laser oriver ( $\$ 150 / \mathrm{J}$ tirect costs). The cost of electricity (COE) for any power plant is: 


$$
\operatorname{COE}=\frac{\mathrm{RC}_{\mathrm{TOT}}+M+F}{8760 \Gamma_{n^{\alpha}}}\left(\$ / \mathrm{kW}_{e} \cdot \mathrm{h}\right),
$$

where

$$
\begin{aligned}
& R=\text { annual fixed charge rate on the capital investment, } \\
& M=\text { annual operation and maintenance cost, } \\
& F=\text { annual fuel cycle cost, } \\
& P_{n}=\text { the net electric power, } \\
& a=\text { availability factor. }
\end{aligned}
$$

Most studjes of fusion power assumed that $M=0.02 C_{\text {Tor }}$ and, for simplicity, we shall do the same. For fission and coal, $F$ will be a 5 ignificant portion of the COE (approximately $25 \%$ for fission and $50 \%$ for coal). Since we have included the capital costs of the target factory in $c_{\text {TOT }}$, the fuel cycle costs for an ICF plant are small ( $2 \$ 0.01 /$ target for materials), and we will neglect them. Thus, we find

$$
\operatorname{COE} \approx \mathrm{C}_{\mathrm{TOT}} / \mathrm{P}_{\mathrm{n}}
$$

We can relate $P_{g}, E_{d}$ and $P_{n}$ to one another through the plant power balance equation:

$$
P_{n}=P_{g}-P_{a}-P_{d}
$$

where

$$
\begin{aligned}
& P_{a}=\text { auxiliary power }=f_{a} P_{g}, \\
& P_{d}=\text { driver power }=E_{d} \omega_{d} / n, \\
& \omega_{d}=\text { driver pulse rate }(H z), \\
& n=\text { driver efficiency. }
\end{aligned}
$$


Also, note that

$$
P_{g}=P_{d} n G M E \text {, }
$$

where

$$
\begin{aligned}
& G=\text { target gain, } \\
& M=\text { energy multiplication due exothermic neutron reactions, } \\
& E \text { = thermal to electric conversion efficiency. }
\end{aligned}
$$

Thus,

$$
P_{g}=P_{n}\left(1-f_{a}-\frac{1}{\eta G M E}\right)-1
$$

The fusion cycle gain, nGMe, just describes the complete set of physics and engineering variables that will determine the recirculating power fraction (with a small correction for $f_{a}$, the auxiliary power). Combining equations 6,1 and 2, we can find an expression for the fusion cycle gain required at any driver energy $\left(E_{d}\right)$ in order to obtain a given COE for any specified net power $P_{n}$. Fractional increases in any of the separate factors of the fusion cycle gain will obviously have the same effect on the recirculating power fraction. However, since some costs scale as $P_{g}$ and others as $E_{d}$, the overall effect on COE will depend on the value of $E_{d}$ considered.

Let us consider, for example, the gain (G) as a function of driver

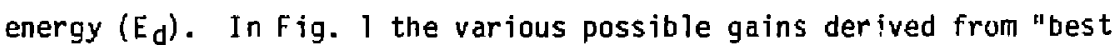
estimate" and "advanced concepts" target calculations are shown. The width of the "best estimate" band represents the range of uncertainty in calculating gain for early power plants. The "advanced technology" curve represents physically possible goals for the longer term future. On the same graph are drawn curves of constant $\operatorname{COE}$ (and $\mathrm{C}_{\mathrm{TOT}}$ ) for $\mathrm{P}_{n}=1 \mathrm{GW}_{\mathrm{e}}$, $a=0.7, f_{a}=0.05$, and the other fusion gain variables of 
$n=0.1, M=1.15$, and $\varepsilon=0.4$. These curves use the same $s \in t$ of economic assumptions as were used in References 1 and 2 and the cost scaling of Equation (1). Thus, we can compare the COE curves to the projected COE of $3.7 \mathrm{k} / \mathrm{kW}_{\mathrm{e}} \mathrm{h}^{\mathrm{h}}$ for future fission and $4.6 \mathrm{k} / \mathrm{kW}_{\mathrm{e}} \mathrm{h}$ for future coal plants. Figure 1 shows that if target design work on the high-gain test faci]ity eventually allows access to the cross-hatched region, then a $C O E$ of less than $5 \mathrm{~d} / \mathrm{kWe}$ *h will be realized. For a given gain curve the minimum COE (and, therefore, the best operating point) will obviously occur for a cost curve that is just tangent to the gain curve. Clearly, the most effective improvement in the gain curves 〈as far as COE and $C_{T O T}$ are concerned) wOuld be to increase gain at the low driver energies. Another piece of information showl in Fig. 1 is the required pulse rate at various intersections of the two sets of curves. The higher the pulse rate, the more difficult it will be to design an acceptable reaction chamber. The lowest cOE will be obtained at a pulse rate of about $10 \mathrm{~Hz}$ for any given $\mathrm{si} i \mathrm{r}$ curve. However, due to the "lens" shape of the available operating space, the dependence is not strong for pulse rates above about $5 \mathrm{~Hz}$.

Obviously, the same type of analysis can be used to explore ather design and cperating spaces. It is encouraging to $\mathrm{s}^{\circ}$ intarize the information in Fig. I by noting that if a high-energ; laser with $10 \%$ efficiency can be built for $\$ 150 / \mathrm{J}$, then laser fusion power is likely to be competitive with future fission and cnal plants at the $1 \mathrm{GW}_{e}$ size. If higher efficiencies or lower costs are achieved (or if the energy conversion efficiency and/or ott or fusion cycie parameters are improved), then ICF can be competitive at even smailer plant sizes.

One of the methods being considered to make fission power more attractive is to build the plant in modules. Given the usual economics of scale, one might think that this will always result in a higher COE. However, there are several factor that may mitigate (or even reverse this effect). First, if the plant modules are small enough to have a 


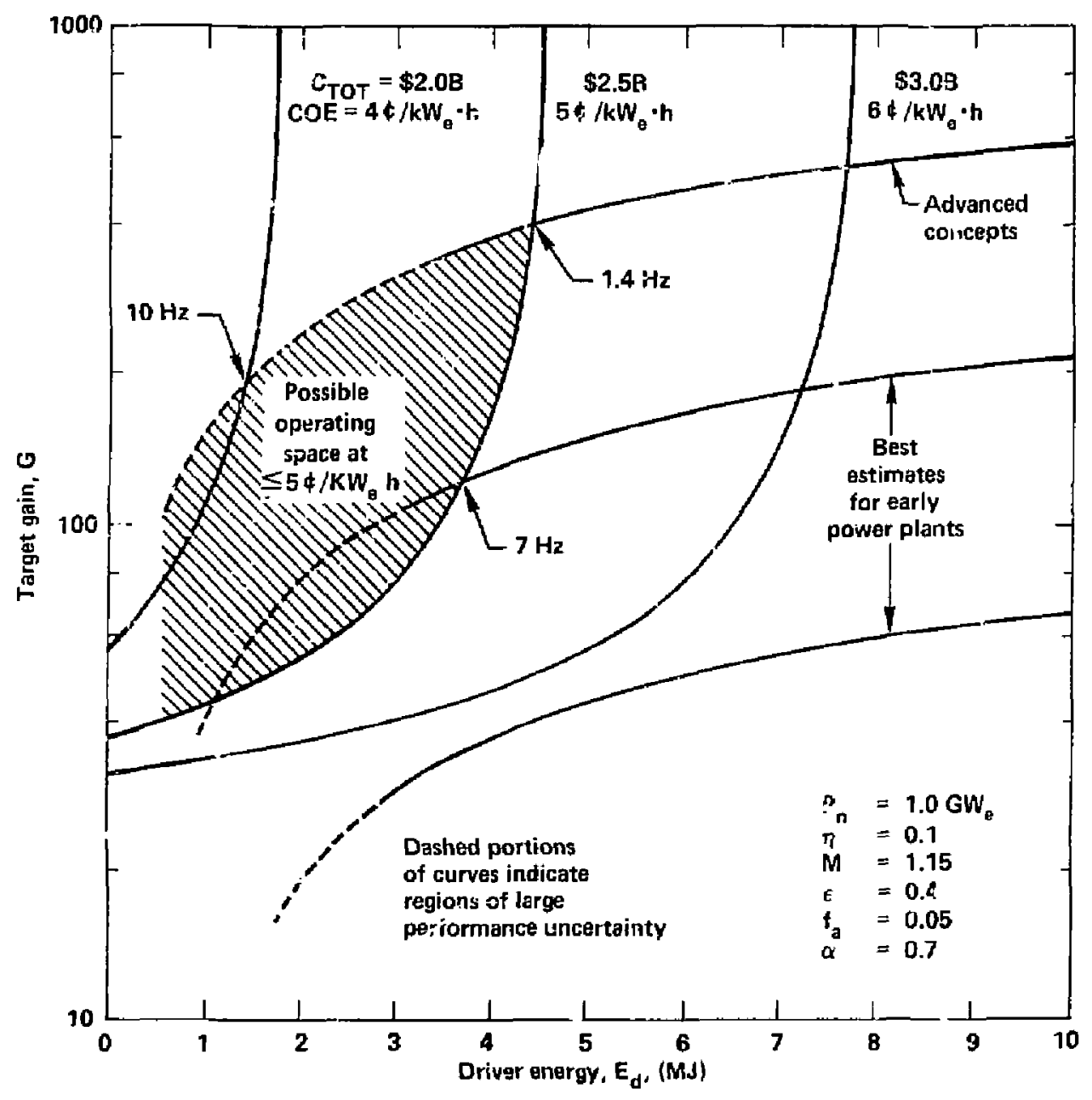

Fig. 1. Curves of Target Gain Obtainable (Physics Calculations) and of Target Gain Required for Certain Cost of Electricity (Economic Calculations) 
greater fraction of the work done in factories rather than in the field, and/ar if the construction time can be shortened, then costs can be reduced. On the other hand, even if costs per unit power are increased, the modular construction may give added decision making flexibility. Projections of demand would not have to be made so far into the future and it might be possible to derive revenue from the first module while the next is under construction.

ICF is particulariy adaptable to modular construction because of the ability of a driver to service more than one reaction chamber. Fig. 2 shows the results of our analysis for module sizes of $0.5 \mathrm{GW}_{\mathrm{e}}$, $1.0 \mathrm{GW}$, and $2.0 \mathrm{GW}_{\mathrm{e}}$. For these curves, the upper "best estimate" gain curve and a pulse rate corresponding to the lowest COE were used. Fig. 2 shows that the COE is high for a single $0.5 \mathrm{GW}_{\mathrm{e}}$ plant, but as further power units are added to the same driver, the COE drops rapidly. The result would be even better if credit were taken for reduced construction time and factory instruction.

\section{Design of the Reactor}

The functions of the reaction chamber are to:

1. Contain the energy and debris from the fusion pulses and protect the outside wor]d.

2. Convert the pulsed $x$ ray, neutron and debris energy into a steady flow of energy in a form than can be easily converted to electricity (or better yet, to convert directly).

3. Breed new tritium to replace that burned.

While doing the above, it is also desirable that: 
$-12=$

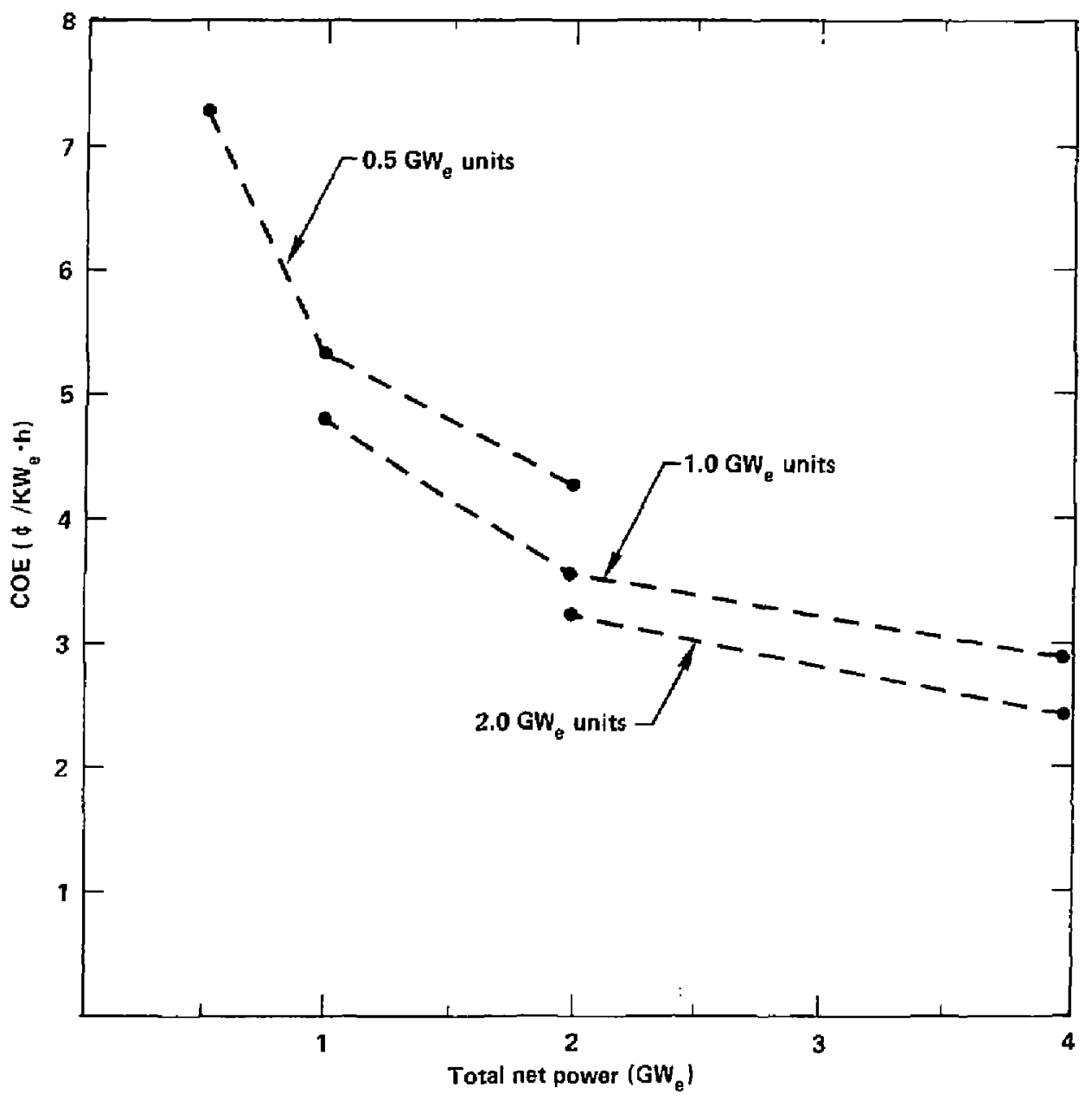

Fig. 2. Cost of Electricity for Modularly Constructed ICF Power Plants 
o Hard to replace structural elements last the lifetime of the plant.

- The inventory of radioactive material (tritium and neutron induced activity) and the routine leakage are kept to a minimum.

- The combination of flammable ard radioactive material is minimized.

o Structural melt due to afterheat in an accidental shutdown is eliminated without requiring any active emergency cooling system (often called inherent safety).

- The biologica 1 hazard potential (BHP) in accidents is minimized.

o The radioactive wastes are low level and short lived (satisfying shallow land burial criteria if possible).

o Hands on maintenance is allowed as murih as possible.

The above goals are the same for a magnetic fusion reactor However, in ICF the physics of beam energy absorption, implosion, ignition, and Durn of the fusion plasma are almost completely separable from the chamber environment and structural requirements. Things happen in series, albeit on very short time scales; interfacing between systems is constraining only at certain times in a fusion pulse cycle. Therefore, there is an extraordinary degree of design and engineering flexibility. One of the major manifestations of this flexibility in ICF reactors is the ability to put flowing materiais (solid granules, liquids, or gases) inside the reaction chamber to protect the structural elements and to take greater advantage of the high quality of fusion energy.

This design flexibility is manifested in the 37 reactor studies that have been done in the last 14 years. The interfaces between various types of drivers (light ions, heavy ions, and several laser types) and the reaction chamber have been studied. The reactor impiications of using directly or indirectly driven targets have also been investigated. Reaction chamber concepts have included the foltowing: 
1. Dry wal1--1inited to low yield with structural elements protected principally by distance, sometimes on inner high temperature layer provides thermal inertia.

2. Gas filled cavities--protects walls from low-energy $x$ rays and debris, thus minimizing vaporization of wall material.

3. Magnetic field protection--diverts charged debris from walls and may provide some direct energy conversion.

4. Thin 7 iquid films--provides self-renewing inner layer to prevent wall vaporization from $x$ rays and debris.

5. Liquid sprays--similar to 4 , but also rejuces effect of ablatively generated shocks.

6. Thick 1 iquid falls and sprays--protects against neutrons as well as $x$ rays and debris; tritium breeding done inside chamber.

7. Thick flowing granular blanket--similar to 6 but can operate at high temperature.

In recent years at LLNL, we have concentrated our studies on thick liquid or granular blankets because of their advantages in extending structural wall ifetime and reducing neutron activation. These designs are most suitable for laser or heavy ion beam drivers and indirectly driven targets. Three such designs will be described in more detail.

\section{A. HYLIFE: A Liquid Lithium Reactar}

In the HYLIFE chamber (shown in Fig. 3), $20 \mathrm{~cm}$ diameter jets of liquid lithium fall in a cylindrica! array between the target and the first structural wall $1^{3}$. The array is 1.5-2.0 m thick with an average 
density about half that of liquid lithium. The lithium fall protects the structure from the effects of the fusion pulse, breeds tritium, and acts as the primary heat exchange medium. The pool of lithium at the bottom and a spray across the top protects structures in these areas.

Targets are injected at a rate of $1.5 \mathrm{~Hz}$, imploded by a $4.5 \mathrm{MJ}$ oriver, and yield $1800 \mathrm{MJ}$ of energy. The 1 ithium exit temperature is $770 \mathrm{~K}$, and the plant power is $3170 \mathrm{MW}_{t}$ and $1010 \mathrm{MW}_{\mathrm{e}}$ (net).

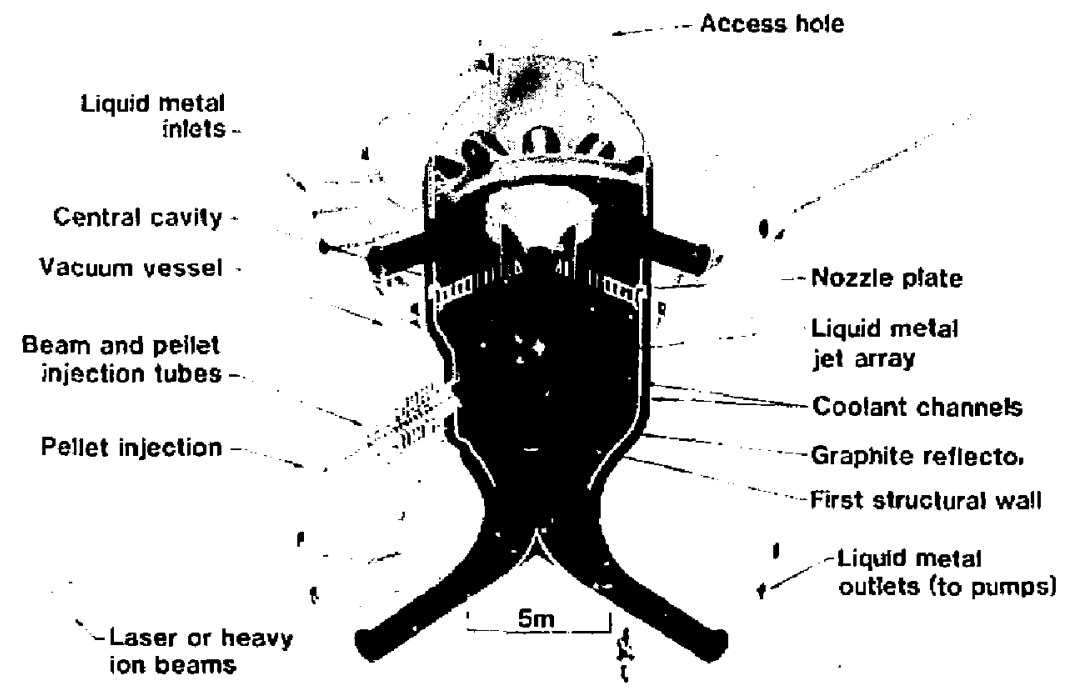

Fig. 3. HYLIFE: A Liquid Lithium Protected Reactur

The flowing lithium jet system is simple in concept and very effective. The 0.75 to $1.0 \mathrm{~m}$ (effective thickness) layer of liquid lithium reduces the $14 \mathrm{MeV}$ neutron flux on the wall by a factor of 200. A common low alloy ferritic steel $(2.25 \mathrm{Cr}-1 \mathrm{Mo})$ is used for the structure; Fig. 4 shaws the lifetime of the wall as a function of the 
lithium thickness used. As can be seen, the wall can be made to last the lifetime of the plant if a thickness above about $0.75 \mathrm{~m}$ is used. Arranging the falling lithium in an array of $20 \mathrm{~cm}$ diameter jets, rather than in a continuous fall, provides effective shock isolation from the fusion pulse. Since the hot gas from vaporized lithium mere: $y$ blows through the array of jets, this configuration minimizes the wall stress due to the impact of lithium accelerated ay high-pressure blowoff gas, caused by $x-r a y$ and debris energy deposition. A substantial fraction of the kinetic energy of expansion of the fluid, resulting from the neutron absorption, is dissipated in the Tiquid-liquid interactions amorg colliding jets. Finally, the enormous surface area of the disassembled fluid acts as an effective condensation pump for the lithium vapor.

In addition to its advantages, the HYLIFE design also raises some issues. The lithium jets must reestanlish themselves between pulses.

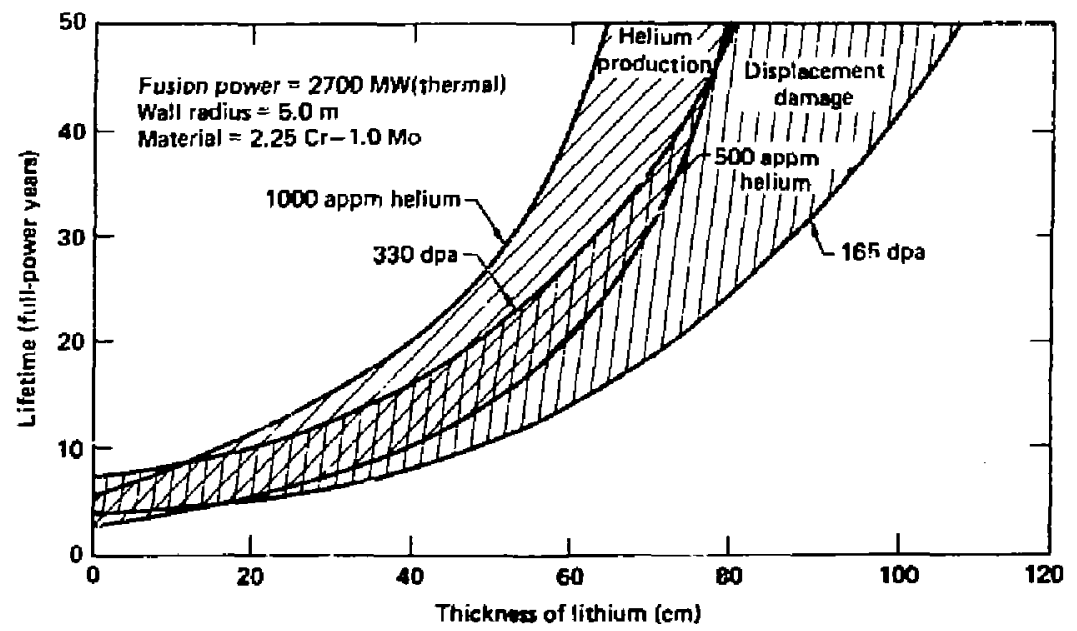

Fig. 4. The lifetime of a $2.25 \mathrm{Cr}-1$ Mo wall located at $5 \mathrm{~m}$ as a function of the thickness of 1 iquid protection. 
Above about $1.5 \mathrm{~Hz}$ the pumping power becomes excessive. As we have seen, this is not optimal for the general economics. The practical pulse rate could be even further limited if it is found that droplets from the disassembling jets often take unfavorable trajectories. At this pulse rate (and, therefore, lithium flow rate), the lithium temperature rise per pulse is only $18 \mathrm{~K}$. The flow rate to obtain the $1.5 \mathrm{~Hz}$ puise rate is, thus, five times that needed for heat transfer. The pumping power is high. Liquid lithium is flammatle and reacts with water. The stored chemical energy in the inventory of 7 ithium is about $70^{13}$ joules (about the same as the sodium in an LMFBR). For comparison, the chemical energy in the railcar of propane is about $10^{12}$ joules. An intermediate neat exchange loop using liquid sodium must be used to separate the tritium bearing lithium from the water in the steam cycle. Furthermore, all concrete must be lined with steel and the reactor rom segmented to minimize lithium-concrete reactions. These factors have combined to make the HYLIFE design about a factor of two more expensive than a LWR.

\section{B. Pulse*Star: A Lithium/Lead Pool-Type Reactor}

The Pulse*Star reactor ( $\mathrm{Fig}$. 5) consists of a pool of $\mathrm{Li}_{17} \mathrm{~Pb}_{83}$ in which a bell-jar shaped reaction chamber is submerged ${ }^{4}$. LiPb sprays through orifices in the bell $\mathrm{j}$ ar and impinges on a metal screen creating a $1.5 \mathrm{~m}$ thick region of droplets at half density that absorb the effects of the fusion pulses, breeds tritium and acts as the heat transfer medium. The screen is protected from $x$ rays and debris by a thin film of $L i P b$ that penetrates the screen and flows down the inside. Pumps and heat exchangers are submerged in the LiPb pool between the reaction chamber and the pool wall.

A higher pulse rate is achjevable in f'uise*Star than in HYLIFE. The repetition rate is limited by the time required to recondense the vaporized LiPb to the level necessary for beam propagation. We estimate that a few $\mathrm{Hz}$ repetition rate can be obtained for a laser oriver. One 


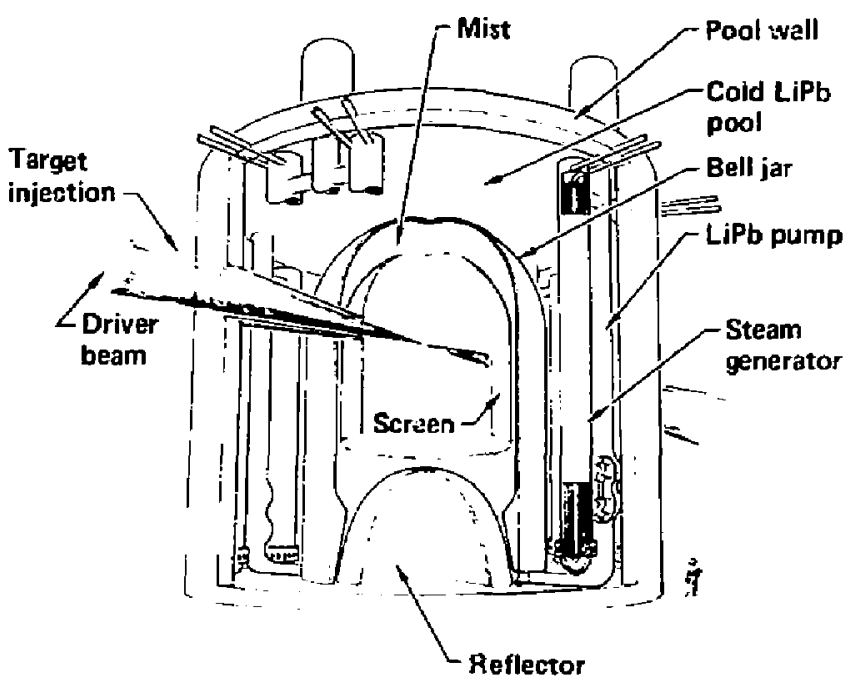

Fig. 5. PulsexStar: A Pool-Type, Lipb Cooled Reactor

Pulse*Star point design uses a $2 \mathrm{MJ}$ driver, a target yie?d of $600 \mathrm{MJ}$ and a pulse rate of $4.3 \mathrm{~Hz}$ to lead to a LiPb exit temperature of $770 \mathrm{~K}$, a gross thermal power of $3300 \mathrm{MW}_{t}$, and a net electric power of $1115 \mathrm{MW}_{\mathrm{e}}$.

Advantages of the Pulse*Star concept are its compact size and the reduction in the number of pipes and valves. The power density inside the pool is 29 times that of a PWR containment building. The use of $\mathrm{LPb}$ eliminates the fire hazard. These factors and the higher pulse rate should lead to lower cost power than for HYLIFE. 
The Pulse*Star design has not been studied as thoroughiy as HYLIFE and has its own set of issues. It is clear that design of the screen will be difficult. It must prevent LiPb dropiets from ralling into the beam lines, must withstand the shock created by vaporization of a few microns of $\mathrm{LiPb}$ on the inside surface, and must allow the hot vapor to pass freely so that it can be recondensed on the numerous droplets between the screen and the bell jar. It is not protected from neutrons so we expect high activation and displacement damage. It will have to be replaced periodicaliy but is a fairly simple structure. The use of Lipb seems to solve the fire hazard and, thus, we have no intermediate coulant loop; however, tritium is very insoluble in L $\mathrm{IPb}$. This leads to a very low tritium inventory (20 gms in the entire pool) but concern has been raised about the routine leakage of triti.m through the large area available in the stean generators. If no precaltions are taken, about $50 \mathrm{~g}$ per day may ciffuse into the water in the steam cycle. Research is underway (for the MCF program) on methods of containment including double-walled heat exchangers with an oxygen sweep gas. The second issue with using the LiPb, to be assessed below, is the creation of $210 \mathrm{Po}$ by neutron activation in the $\mathrm{Pb}$.

\section{Cascade: A Centrifugal-Action Ceramic Li Granule Reactor}

Structural elements of the cascade reactor (Fig. 6) are protected by a $1 \mathrm{~m}$ tnick layer of solid Li ceramic granules that are about the size of grains of sand 5 . These granules, injected into the two ends, flow through the $4.5 \mathrm{~m}$ radius chamber, and are held against the wall by rotating the entire chamber at about $0.8 \mathrm{~Hz}$. The kinetic energy acquired from the chamber is also used to throw the granules into the vacuum heat excnanger. They return to the reaction chamber by gravity flow. Use of $L i$ ceramic granules allows operating at high temperatures to obtain a high tinermal to electric conversion efficiency. The wall consists of SiC tiles hela in compression with a network of $\mathrm{Ai} / \mathrm{SiC}$ composite tendons. A particularly abrasion resistant material, SiC tiles are now in use in 
printing presses. Like any ceramic material, SiC is nct good in tension and, therefore, the tiles are held in compression even during the fusion pulse. Two blanket designs have been considered to date. In one a uniform blanket of $\mathrm{Li}_{2} \mathrm{O}$ is used at an exit temperature of $1200 \mathrm{~K}$ (limited by chentcal compatibility issues raised by the formation of LOOT). In the other, a two-layered blanket is used. $L \mathrm{LA} 10_{2}$ is used in the tritium-breeding outer layer, and BeO is used as a neutron multiplier and high-temperature inner layer. In this latter design, the layers keep themselves separate if different size and density are chosen for the different granules. The mean-exit temperature of this blanket is $1670 \mathrm{~K}$, but the inner layer of Be0 reaches $2300 \mathrm{~K}$. One point design with the two-layer blanket uses a $1.5 \mathrm{MJ}$ driver operating at $5 \mathrm{~Hz}$ with fusion pulses of $300 \mathrm{~mJ}$. With a Brayton cycle, this design coulo attain a thermai to electric conversion efficiency of over $55 \%$. This design would praduce a net power of $800 \mathrm{MH}_{\mathrm{e}}$ with a thermal power of only $1670 \mathrm{MW} t$.

The greatest advantages of this concept are its potentially high energy conversion efficiency and its high pulse rate. Even though a vacuum heat exchanger is used, the very high temperatures result in good heat transfer efficiency and, thus, nominat required heat transfer area (and cost). The pulse rate of cascade is determined by the recondensation of vaporized material. With each fusion pulse, about $1 \mathrm{~kg}$ of material is vaporized. While there is a great dea? of uncertainty about the phenomenology of energy and mass transfer between a not, ionized plasma and a cold wall, the tremendous surface area represented by the granule bed makes it likely that the recondensation will take place in less than $100 \mathrm{~ms}$. Cascade also has low auxiliary power requirements and a low fire hazard. These factors can all lead to more economic power.

Several issues are of concern for Cascade. The inner layer of granules may comminuie (break apart) due to $x$-ray and debris spall or thermal stress. On the other hand, the recondensing vapor may cause some 


$$
-21-
$$

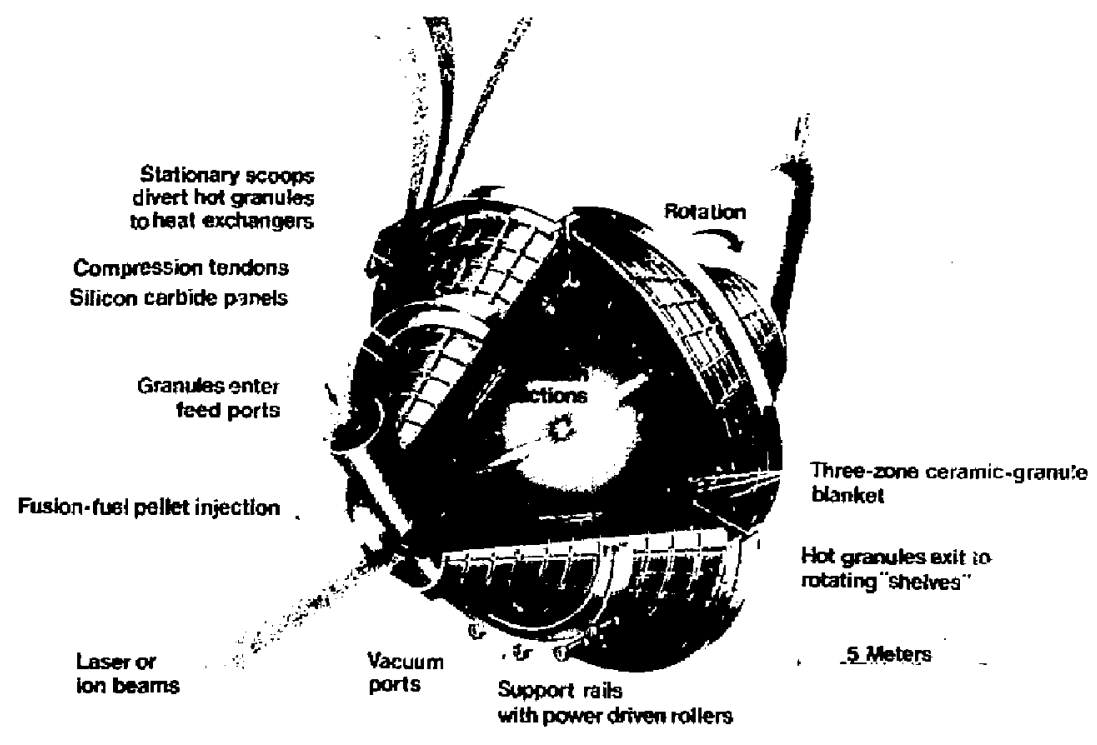

Fig. 6. Cascade: A Rotating-Ceramic-Granule-B lanket Reactor

granules to aggragate. It is not clear that the granule manufacturing process will be inexpensive enough that this layer can be remanufactured with each pulse. The practicality of operating at such high temperatures must also be considered. Finally, the recavery and recycling of the lead from the target debris must be addressed.

D. The Iriver-Reaction Cnamber Interface

A11 three reaction chamber concepts were designed for two-sided illumination by either lasers or heavy ion beans. From the reactor design standpoint, it would be desirable $\because \because$ have as few penetrations with 
as small a solid angle as possible. The present designs assume that the $f$ inal laser mirrors are several tens of meters away from the fusion pulse (60 $\mathrm{m}$ for HYLIFE). The mirrors are protected from the effects of sof $t$ $x$ rays and debris by a few torr-meters of a high $Z$ gas in one section of the beam pipe. The critical damage may come from the neutrons and that is why distance may be the only solution. It is estimated that the final mirrors for HYLIFE would survive for about one year and would then have to be replaced. Upstream from the final mirrors, and out of the direct line of sight from the fusion pulse, the beams would enter the containment building through a large window (with a backup-shuttering system).

For laser propagation through the chamber to the target, the nominal atom density to avaid breakdown should be below about $10^{15}-10^{16} \mathrm{cmi}^{-3}$ (two to three orders of magnitude below the peak density attained). All of the designs considered appear capable of recondensing the vaporized material to this level in times of tise order of tens of milliseconds. On the other hand, heavy ion beam propagation may require a number density of $10^{12}-10^{13}$ (unless ane of the high-pressure transmission "windows" is found to be usable). For this large a density change (five or six orders of magnitude), the uncertainties on recondensation phenomena loom more ominously. Furthermore, use of some blanket materials is difficult. Far example, the vapor pressure of lithium at $770 \mathrm{~K}$ is $210^{-2}$ torr. To use heavy ion beam with the HYLIFE design would require lowering the lithium temperature to $720 \mathrm{~K}$, using $\mathrm{LiPb}$ instead, or perhaps injecting cooler lithium near the beam lines to reduce the number density locally.

The narrow, two-sided illumination discussed above is not optimal from the target design standpoint (even for indirect-drive targets). There is a penalty to pay in terms of increased driver energy if all beams approach the target from only two directions. Calculations indicate that this penalty is quite modest. However, to assess the 
impact on reactor design, let us consider illumination by eight beams (four on each side at an angle of $30^{\circ}$ from the horizontal). The HYLIFE and Pulse*Star designs could probably accommodate such an eight-beam arrangement with relative ease. In Cascade it would be more difficult because of the rotation of the chamber. If there were eight-beam ports in the sides of the Cascade chamber (four on each end), the granules could conceivably flow around them. The timing of the chamber rotation would have to be such that the ports lined up with the beam lines each time the laser was to be fired. Since the rotation rate best for granular flow is near to that necessary for port alignment, perhaps this is not too far fetched. This tradeoff certainly bears more examination.

\section{E. Safety and Environmental Issues}

An indication of the size of the radioactivity problem is shown in Tahie I for five reactors. In addition to the induced activity of the three ICF reactors discussed in this paper, the total radioactivity in a typicai pressurized water reactor (PWR) and the induced activity in the Starfire magnetic fusion reactor are shown for comparison. It is seen that the Cascade with an $L_{1} i_{2} 0$ blanket not only has the smallest amount of activity at shutdown, but its activity is short lived. In fact, at so years the activated material from Cascade would satisfy U. S. government standards for shallow land disposal without dilution. It is the coly one of the five reactors for which this is true.

Decay afterheat shown in Table II is an indicator of the need for emergency cooling to prevent meltdown. If values are smaller than a few $M W$, it is likely that radiative cooling would be sufficient and no active cooling system would be required. Both Cascade $\left(\mathrm{Li}_{2} \mathrm{O}\right)$ and HYLIFE satisfy this criterion at shutdown and again Cascade's value falls rapidly after shutdown.

Finally, the inhalation biological hazard potential (BHP), an indicator of the potential risk due to release of debris to the 
Table I. Tota? neutron induced activity ( $\mathrm{kCi}$ ) for a $1000 \mathrm{MH}$ reactor operating 30 jears.

Time afte' shutdown

\begin{tabular}{|c|c|c|c|c|c|}
\hline \multirow[b]{2}{*}{ Reactor Type } & \\
\hline & $\underline{0}$ & $1 d$ & $1 \mathrm{~m}$ & $70 y$ & $100 y$ \\
\hline PWR & $1.7\left(10^{7}\right)$ & (a) & (a) & (a) & (a) \\
\hline Starfire & $6 \quad\left(10^{6}\right)$ & (a) & (a) & 2400 & 292 \\
\hline HYLIFE & $3 \quad\left(10^{5}\right)$ & $\left(10^{5}\right)$ & $1.5\left(10^{5}\right)$ & 90 & 33 \\
\hline Pulse*Star & $\left(10^{6}\right)$ & $\left(10^{5}\right)$ & $7 \quad\left(10^{5}\right)$ & 660 & 220 \\
\hline Cascade $(1 ; 20)$ & $2 \quad\left(10^{5}\right\}$ & $7 \quad\left(10^{3}\right)$ & $6 \quad\left(10^{3}\right)$ & 11 & 8 \\
\hline Cascade $\left(\right.$ LiA $\left.10_{2}\right)$ & $1.1\left(10^{6}\right)$ & $7.7\left(10^{4}\right)$ & $5.4\left\langle 10^{2}\right\rangle$ & 26 & 22 \\
\hline
\end{tabular}

(a) not available

biosphere, is shown in Table III. It is the volume of air that must be mixed with the radioactive material in order to make it safe for humans to breath. Of course, any given accident would release only a small fraction of the material but, again, the total amount is used as a relative indicator of the magnitude of the problem. Cascade $\left(\mathrm{Li}_{2} \mathrm{O}\right)$ again appears to hove the lowest potential $r$ isk.

Two facts stand out in these comparisons: 1) all ICF reactors appear to be better than either a PWR fission plant or the Starfire magnetic fusion design for these figures of merit, and 2) the Cascade $\left(L_{i} i_{2}\right)$ reactor is superior to the other ICF designs. The $L \mathrm{JA10} \mathrm{O}_{2}$ version of Cascade was not optimized to reduce activation prociucts. A more complete design study is in progress.

\section{ICF Hybrid Reactors}

As with MCF, there is another moje in which ICF reactors can help to supply power. They can produce the fuel burned in fission reactors. 
Table II. Decay heat of induced activity (kH) for a $1000 \mathrm{MW}$ reactor operating 30 years.

Time after shutdown

\begin{tabular}{|c|c|c|}
\hline \multirow[b]{2}{*}{ Reactor type } & \multirow{2}{*}{\multicolumn{2}{|c|}{$1 d$}} \\
\hline & & \\
\hline PWR & $1.8\left\langle 10^{5}\right\}$ & (a) \\
\hline Starfire & $7\left\langle 10^{4}\right\}$ & (a) \\
\hline HYLIFE & $2\left\langle 10^{3}\right\rangle$ & $2 \quad\left(10^{2}\right)$ \\
\hline Pulse*star & $1.8\left\langle 10^{4}\right\}$ & $1.4\left(10^{3}\right)$ \\
\hline Cascade $\left(\mathrm{Li}_{2} \mathrm{O}\right)$ & $8 \quad\left(10^{3}\right)$ & Tt \\
\hline Cascade $\left(\mathrm{LiA} \mathrm{IO}_{2}\right)$ & $2.3\left(10^{4}\right)$ & $2.1\left(10^{3}\right)$ \\
\hline
\end{tabular}

(a) not available

Table III. Inhalation bhp $\left(\mathrm{km}^{3}\right)$ for $1000 \mathrm{MW}_{\mathrm{e}}$ reactor operating 30 years.

Time after shutdown

\begin{tabular}{lll} 
Reactor type & 0 & $1 \mathrm{~d}$ \\
\hline PWR & $1.2\left(10^{10}\right)$ & (a) \\
Starfire & $1.7\left(10^{10}\right)$ & $(\mathrm{a})$ \\
HYLIFE & $5\left(10^{7}\right)$ & $3\left(10^{7}\right)$ \\
Pulse*Star & $7\left(10^{8}\right)$ & $6\left(10^{8}\right)$ \\
Cascade $\left(\mathrm{Li}_{2} 0\right)$ & $8\left(10^{6}\right)$ & $1.3\left(10^{6}\right)$ \\
Cascade $\left(\mathrm{LiA}_{2}\right)$ & $7.4\left(10^{7}\right)$ & $1.5\left(10^{7}\right)$
\end{tabular}

(a) not available 


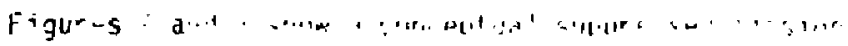

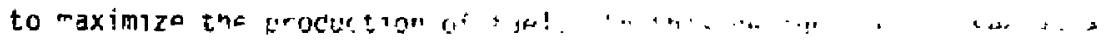

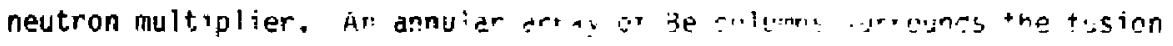

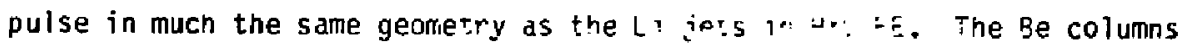
consist of short cylinders of Be joined together with steel snap rings. Vertical holes in the Be carry lithium coolant and thorium wires that breed ${ }^{233} \mathrm{U}$ and tritium. The columns are loose fitting so that some lithium will leak out. That is of no consequence since the columns are further protected from the $x$-ray and debris energy by a thin 1ithium fall in the chamber. One of the critical issues in this hybrid design is the radiation damage effects in Be.

The fusion breeder has a tremendous advantage over a fission breeder. The number of neutrons available per unit thermal energy is several times larger. Each fusion reactor can support up to 18 fission reactors of comparable size. The ICF hybrid also holds a smalt advantage over MCF hybrids because the breeding material can be exposed more directly to the neutrons inside the reactor. This leads to about a $20 \%$ advantage in neutrons per unit thermal energy. One point design of the ICF hybrid has a $4 \mathrm{MJ}$ driver, a yield of $400 \mathrm{MJ}$ and a pulse rate of $4 \mathrm{~Hz}$. With a net electric power itself of $1000 \mathrm{MW}$, it will produce $6000 \mathrm{~kg}$ of ${ }^{233} \mathrm{U}$ per year, enough for 18 fission reactors. Even with a capital cost between 1.5 and 2 times that of an LWR, it could produce fuel at a $U_{3} \mathrm{O}_{8}$ equivalent cost of $\$ 30$ to $\$ 40$ per pound.

The high reactor support ratio has several implications that were explored in a recent study ${ }^{6}$. It is not well known when the anticipated fissile fuel shortage will occur (witness the lack of interest in the LMFBR at present), and how severe it will be. Furthermore, past price fluctuations in $U_{3} \mathrm{O}_{8}$ have been large and they have occurred on time scales that are short compared to those necessary for increasing production from mines. The LMFBR would have difficulty responding to such a rapidly changing environment. Development of an ICF breeder would 
allow a more timely response to fuel shortages and would lead to a cap on fissile fuel price escalation.

One final point should be made about ICF hybrids. The same arguments that reveal such an advantage in making fission fuel also apply to making tritium and plutonium for the nuclear weapons stockpile. One tritium optimized design could produce tritium for less than half the anticipated cost than that from a new fissjon-powered production reactor. A similar advantage should apply to plutonium. If an ICF hybrid were chosen in place of a new fission powered production reactor, the government would save several billion dollars in these production costs.

ICF hybrids, in general, do not require as high a gain, pulse rate, or as low a driver cost to be competitive. They might, therefore, represent an earlier opportunity to apply ICF technology to power production.

\section{Development Issues}

There have been a large number of ICF reactor concepts proposed and a large number of studies of ICF reactor issues. The reactor design community (limited as it is) is still filling out the matrix of possible ICF design and operating space. It would be premature to select any one design as the best one at this time. However, all reactor design studies suffer from the lack of applicable data, often even for the most fundamental and generic issues. The ICF reactor design effort has benefitted from the MCF technolcgy pragram. Some of the fundamental issues about use of $L i, L i P b$, ard $L i$ ceramics as blanket materials are being addressed in that program and the results are usually applicable to ICF reactors as weil. On the other hand, data about ICF specific problems are sorely lacking.

Some of the specific areas where data are needed include: 


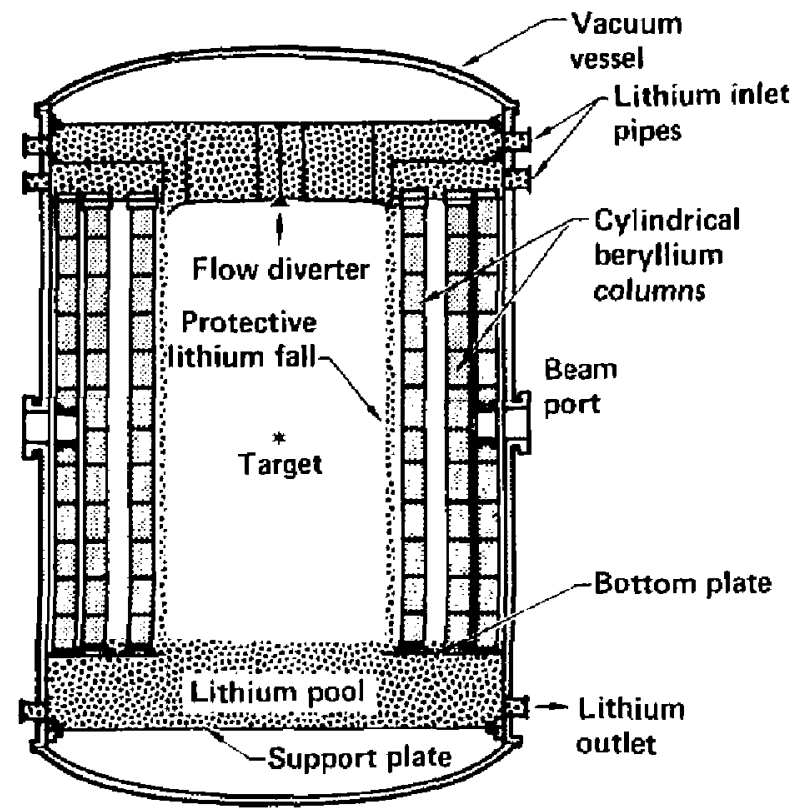

Fig. 7. The chamber design for the TPR uses a Be multiplier and a $L i$ breeder. Adding thorium wire allows production of $233_{U}$.
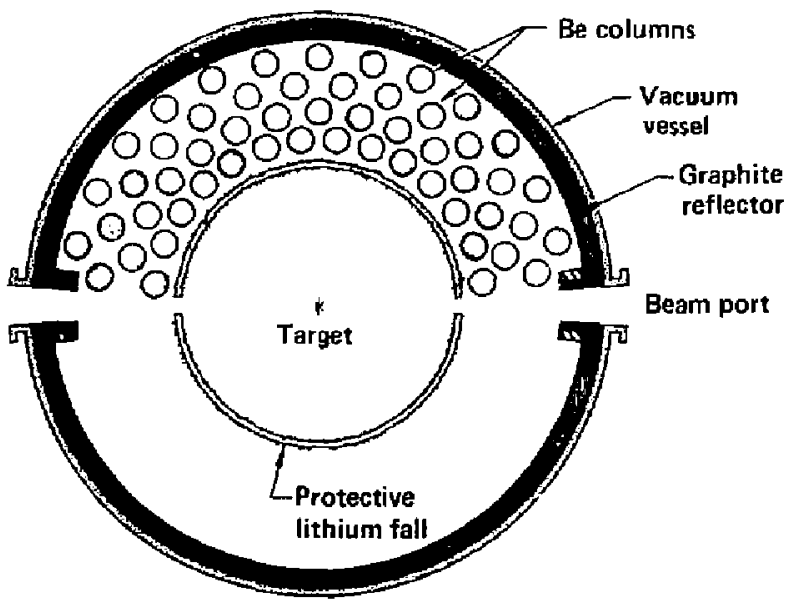

Fig. 8. Cross-sectional view of the Chamber. An array of Be columns surrounds the fusion target. 
1. neutron damage to refiective optics,

2. disassembly of isochorically heated liquid metals,

3. recondensation phenomenology,

4. radiation damage from rapidly pulsed sources,

5. cracking and fracturing of solids in the pulsed radiation environment,

6. and extraction of target materials.

In addition to the data, system concepts and studies are needed on automated production targets $\left(10^{5} /\right.$ day) of ; target injection, pointing and tracking; reprocessing of target materiais; and what containment will mean for an ICF reactor. Finally, ICF will need to create a development scenario that is affordable. For this last item, it appears, again that ICF may have an advantage over MCF. Once high gain is achieved, it is easy to conceive of an inexpensive engineering test facility at low power ( low gain and/ar pulse rate). ICF's engineering tests $c$ an be done at low power by moving the test wall closer to the fusion pulses to obtain any desired flux.

VI. Conclusions

At the same time as progress on drivers and target physics has been proceeding rapidly, so, too, has the study of ICF reactor concepts. The studies to date have shown that ICF reactors can be practical, $c$ lean and safe. Future studies are likely to emphasize econonic competitiveness, small size, and inherent safety. Most past studies have assumed use of the steam cycle. We expect that there may be more emphasis in the future on advanced concepts that convert fusion power into electric power more directly. 
It appears that there are scenarios for economic power production from pure ICF. Further, the ICF hybrid seems to be a desirable option if fission power should experience a resurgence. The accomplishment of high gain appears to be close enough that it is time to begin a serious $1 \mathrm{CF}$ reactor technology program and to increase industry involvement. Obtaining ICF specific data, through fundamental studies at universities and system studies in industry, would be a good first step.

\section{References}

1. J. G. Delene, et al, Nuclear Energy Cost Data Base - a Reference Data Base for Nuclear and Coal-fired Powerplant Power Generation Cost Analysis, U. S. Department of Energy Report, D0E/NE-0044/2 (March 1984).

2. Energy Economic Data Base (EEDB) Program Phase VI Update (1983) Report, United Engineers and Constructors Report, Philadelphia, PA, JUE\&C-ORNL-840630 (June 1984).

3. M. Monsler, et al, Lawrence Livermore National Laboratory 1981 Laser Program Annual Report UCRL 50021-81, pp 8-33 to 8-50.

4. J. A. Blink, et $a^{1}$, Lawrence Livermore Natinr;al Labogatory 1981 Laser Program Annual Repart UCRL. 50021-83, pp 7-29 to 7-40.

5. J.H. Pitts, et al, ibid, pp 7-4 to 7-29.

6. C. E. Max, "Uranium Resources and Their Implications for Fission Breeder and Fusion Hybric Development", Lawrence Livermore National Laboratory, UCRL 90820, May 1984. 